\title{
Interactions of Peptide Coated Gold Nanoparticles with Spike Protein of the SARS-CoV-2: A Basis for Design of a Simple and Rapid Detection Tool
}

\author{
Nitu Verma, Yogesh Badhe, Rakesh Gupta*, Auhin Maparu and Beena Rai \\ Physical Sciences Research Area, Tata Research Development and Design Centre, TCS \\ Research, Tata Consultancy Services, 54B, Hadapsar Industrial Estate, Pune - 411013, India \\ *Corresponding author: gupta.rakesh2@tcs.com \\ Phone : $\quad+91-20-66086422$
}

\begin{abstract}
The outbreak of severe acute respiratory syndrome coronavirus 2 (SARS-CoV-2) has caused a pandemic which not only created a situation of dealing with public health emergency but also triggered the financial crisis of international concern. The current situation demands rapid, convenient and reliable diagnosis of the disease to downregulate its spread. Primary method of diagnosis presently being used, such as nucleic acid testing (RT-PCR), CT scans etc. involve time-consuming advanced machinery for imaging/ RNA replication and highly skilled technicians which could be only done in a laboratory set-up. A rapid, simple yet selective naked eye detection methodology that does not require any advanced instrumental techniques is highly desirable.

In this study, we report computational results which could form the basis of a simple and rapid strategy for the detection of SARS-Cov-2 using peptide (screened from angiotensinconverting enzyme 2 (ACE2) receptor of host cell) functionalized gold nanoparticles (GNPs). This is based on the preferential binding of viral spike (S) protein to ACE2 receptor situated on the surface of the host cell membrane by which the virus gains access to the host cell. The interaction of peptide coated GNPs with spike protein has been investigated using coarse grained molecular dynamic simulations. The potential of mean force calculation of spike protein confirmed strong binding between peptide and receptor binding domain (RBD) of spike protein. The results presented here demonstrate the potential of this peptide coated GNPs-based system in the development of convenient sensors for the clinical diagnosis.
\end{abstract}




\section{Introduction}

COVID-19 pandemic has posed a serious threat against public health and global economies, with nearly 60 million reported infections and over 1.3 million deaths as of Nov, 2020 [1, 2] Characterized by symptoms of pneumonia, the 2019 novel coronavirus (2019-nCov) was identified from the bronchoalveolar lavage fluid of a patient [3]. It has genomic sequence similarity of about $82 \%$ to that of previously known SARS-CoV detected in 2003 , hence it was named SARS-CoV-2 by the International Committee on Taxonomy of Viruses [4, 5]. Specifically, they have two structural proteins, the nucleocapsid and envelope proteins that share $96 \%$ and $89.6 \%$ similarity respectively in terms of genomic sequence [5-7]. Recent experimental studies have confirmed that both SARS-CoV and SARS-CoV-2 specifically bind to the human angiotensin-converting enzyme 2 (ACE2) receptor of the host cell [7-11]. However, SARS-CoV2 recognizes human ACE2 more efficiently than SARS-CoV, increasing the ability of SARS-CoV2 to transmit from person to person and thus is more contagious than SARS-CoV and spreads faster $[7,8]$.

Coronaviruses (CoVs) are largest RNA virus family divided into $\alpha, \beta, \delta$ and $\nu$ genera. Both SARSCoV and SARS-CoV-2 belong to $\beta$ genus with SARS-CoV-2 virions being about $50-150 \mathrm{~nm}$ in diameter containing a positive-stranded RNA $[5,6]$. It has distinctive spikes on surface of about 8 to $12 \mathrm{~nm}$ in length [6]. The viral RNA is protected inside a lipid membrane envelope derived from the host cell $[12,13]$. The viral genome normally encodes four main structural proteins, spike (S), envelope (E), membrane (M), and nucleocapsid (N), as well as several nonstructural proteins and multiple unique accessory proteins [14]. These proteins are responsible for various critical function such as binding of the virus to the host cell receptors, fusion of virus envelop to host cell, fusion of viral RNA inside the host cell and replication of the RNA inside the host cell [15]. The SARS-CoV-2 entry into host target cells is dependent on the binding of homotrimeric spike (S) glycoprotein present on its envelope to a human ACE2 cell receptor and the subsequent priming of the aforementioned protein by proteases (mainly TMPRSS2 and lysosomal cathepsins) provided by host cell. Cleaving and activation of Sprotein is critical for endocytosis or direct fusion of viral membrane to the host cell membrane $[10,16]$. Therefore, spike determines to some extent the host range. 
As stated above, the $\mathrm{S}$ protein is responsible for the virus attachment to the host cell through which virus gains access. Each S-protein monomer has two subunits S1 and S2. Fragment of S1, called receptor binding domain (RBD), undergoes conformational change in order to bind to the ACE2 receptor $[17,18]$. Once bounded, S2 subunit further helps in the fusion of the membrane envelope of the virus to the host cell membrane $[18,19]$. After fusion of the virus to the host cell, the virus RNA is released which undergoes replication and transcription leading to proliferation of virus in the host [20]. Owing to the importance of these two viral proteins, many studies $[3,5,15,21]$ have focused on understanding the preferential binding of S protein to host cell and viral RNA so as detect and inhibit SARS-CoV-2.

At present, nucleic acid testing is the primary method for diagnosing COVID-19. RT-PCR (Reverse Transcription Polymerase Chain Reaction) kits have been designed to detect SARSCoV-2 genetically by reverse transcription and amplification of its RNA into complementary DNA $[22,23]$. Serological tests based on the presence of viral antibodies in the blood of exposed individuals are also being developed to understand the full extent of viral spread [24]. CT scans have been used for clinical diagnosis of COVID-19 in some areas [25]. Other emerging diagnostic tests based on nucleic acid testing (LAMP [26], SHERLOCK [27]) and protein testing (ELISA [28]) have also been reported. All these biosensing techniques mostly require advanced instruments and need of an expert, which are time-consuming and cumbersome as well as susceptible to viral gene mutation [3, 22].

Efforts have been made for the development of simple, reliable and rapid detection methodologies $[3,21,29]$. In this regard, gold nanoparticles (GNPs) have been extensively utilized in many rapid colorimetric biosensing applications due to their exceptional optical properties such as high extinction coefficient, localized surface plasmon resonance, and inherent photostability $[3,29]$. For example, Moitra et al. used GNPs capped with suitably designed thiol modified antisense oligonucleotides (ASOs) specific for $\mathrm{N}$-gene (nucleocapsid phosphoprotein) of SARS-CoV-2 RNA for selective "Naked-eye" detection of SARS-CoV-2 [3]. The thiol modified ASO capped GNPs agglomerate selectively in the presence of its target RNA sequence of SARS-CoV-2 and demonstrate a change in its surface plasmon resonance (SPR) and subsequent color change of the solution (Figure 1(a)). The technique however, required extensive process of viral RNA extraction, isolation and replication. The other important technique was proposed by Seo et al., where they used graphene surface for preferential 
binding of S-protein to its antibody [21]. FET based sensor was attached to the graphene surface for the detection of the virus. The technique also requires complex system setup as shown in (Figure 1(b)).

(a) RNA+ ssDNA coated GNPs

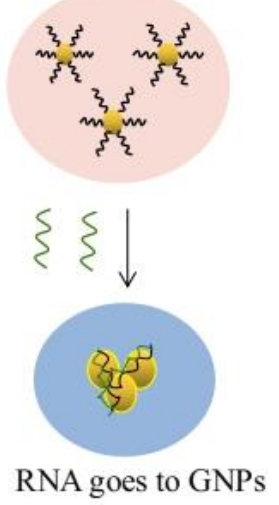

Colorimetric detection RNA Based (b) S protein + Antibody Functionalized Graphene
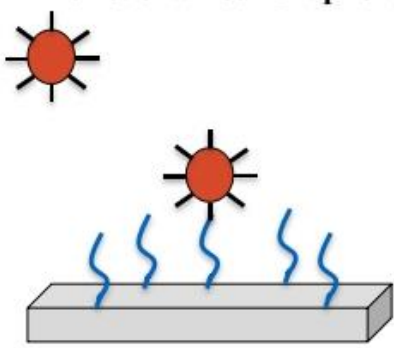

Virus goes to Graphene

FET detection S Protein Based (c) S protein + ACE2 based biomolecule capped GNPs

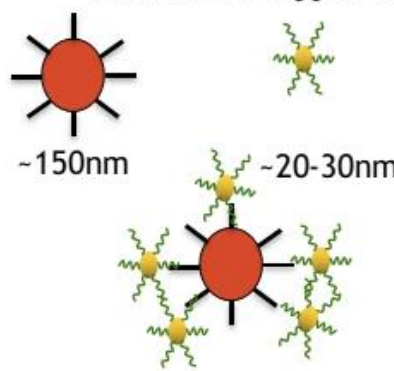

GNPs goes to virus

Colorimetric detection $S$ Protein Based

Figure 1: Schematics for detection techniques for SARS-CoV-2 (a) RNA based colorimetric detection using GNPs [3]. (b) FET based biosensing using preferential binding of $S$ protein to antibody functionalized Graphene [21]. (c) S protein based colorimetric detection using biomolecule capped GNPs (biomolecule: antibody[29], peptide (present study))

In this study, we propose a possible approach for the colorimetric detection of SARS-CoV-2 using GNPs functionalized with peptides having preferential binding to the S-protein (Figure 1(c)) of virus. Due to the plasmonic nature of GNPs, this technique could lead to naked eye detection of the SARS-CoV-2. This type of techniques, based on interaction between biomolecule (obtained from host cell surface receptor protein) functionalized GNPs and viral surface structural protein has been proposed previously for other viruses [30, 31]. Niikura et al. used GNPs coated with sialic acid linked lipids for the optical detection of JC virus like particles [30]. Zheng et al. proposed detection of influenza viruses with glycan-functionalized GNPs [31]. Both the JC virus and influenza virus have spike like entities on their surfaces which interact with receptors of host cells. This preferential interaction of receptors and spike like proteins has been leveraged for the detection in these studies [29, 30]. The colorimetric assay depends on an ordered GNP structure covering the virus surface leading to change in plasmon response. 
Since the S-protein of SARS-CoV-2 interacts preferentially with ACE2 receptors, a peptide derived from ACE2, which interacts with RBD of spike can be used for the detection of the virus. In our recent work, we have screened some of these potential peptides for SARS-CoV2 inhibition based on their stability in aqueous solution and binding affinity to spike protein [32]. We have used one of these potential peptides for functionalizing the GNPs. The potential use of these peptide coated GNPs for the detection of SARS-CoV-2 has been explored in this study using extensive molecular dynamics simulations. The peptide coated GNPs were found to have strong binding affinity towards viral spike protein thereby confirming their possible use in the development of a colorimetric assay.

\section{Materials and Methods}

The Peptide structure (amino acid sequence- EQEERIQQDKRKNENEDKRYQRYGRGKGHQP) was taken from Badhe et al. [32]. All simulations were performed using GROMACS 2018 software [33-35] and visualized using VMD 1.9.1 [36]. The peptide and S-protein were modelled using the MARTINI force field [37]. The peptide was bound to the GNPs through a thiol bond. The coarse-grained (CG) parameters of thiol coated GNPs were taken from previous simulation studies [38-40]. The peptide coated GNP was simulated in the aqueous solution to check the stability of secondary structure of the peptides. Further, agglomeration of peptide coated GNPs is studied to analyze the stability of the system. Finally, binding of spike protein with peptide coated GNPs is observed. The schematic diagram of the present study is shown is

Figure 2.

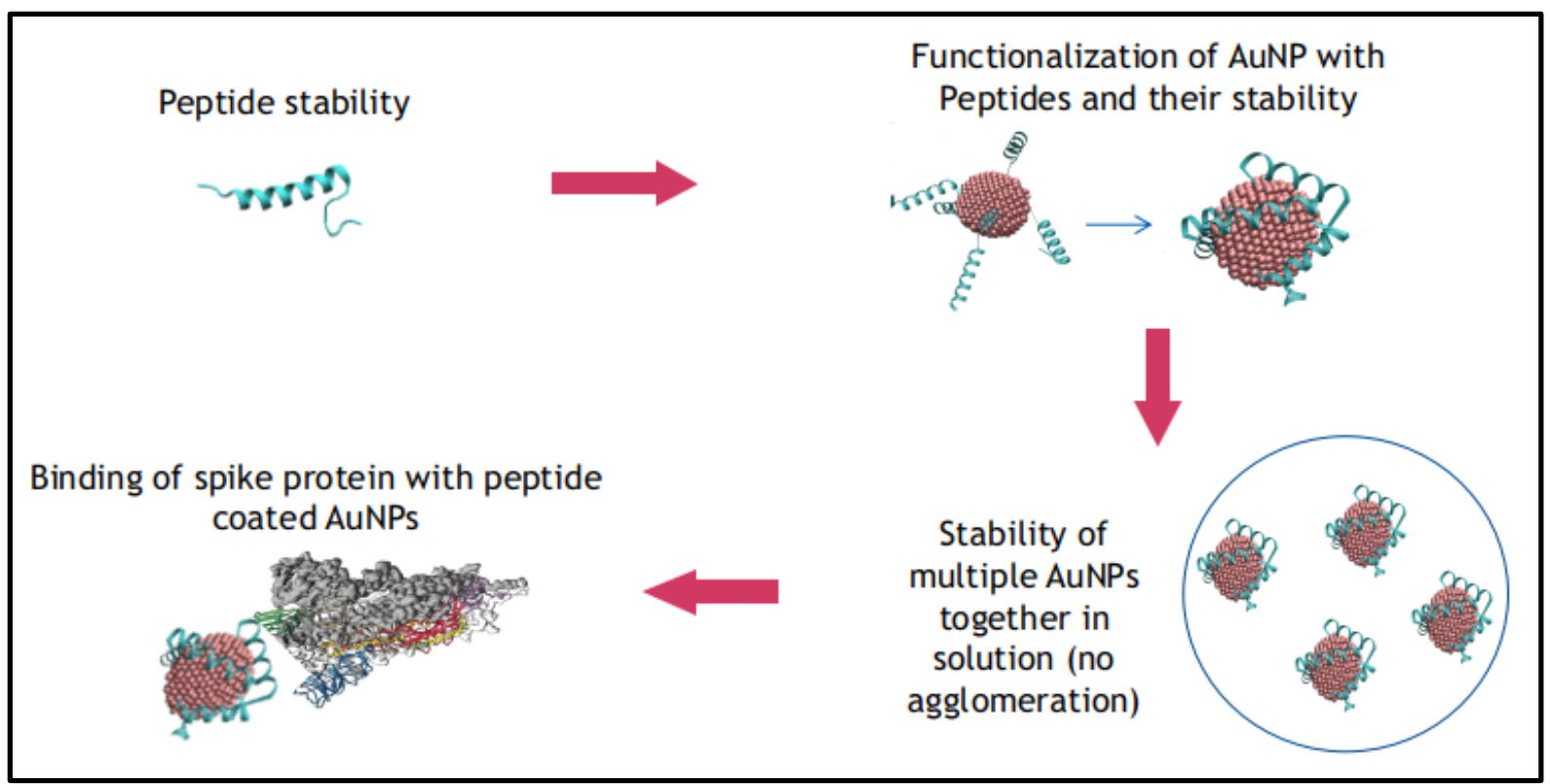


Figure 2: Schematic representation of detection of spike protein using peptide coated GNP. The images are rendered using VMD software.

The peptide coated GNPs and spike were energy minimized individually in vacuum. The structures were solvated with water and ions were added to neutralize the system. Again, the systems were energy minimized and further simulated under NVT (10 ns) and NPT (10 ns) ensemble. During these equilibration runs; the peptide coated GNPs and spike were restrained at their position. These systems were further simulated for $0.5 \mu \mathrm{s}$ at $\mathrm{T}=300 \mathrm{~K}$ and 1 bar pressure. The pressure was coupled isotopically with the compressibility of $4.5 \times 10^{-5}$ bar $^{-1}$. Temperature and pressure were controlled by v-rescale thermostat and ParrinelloRahman barostat with a time constant of 2 ps and 12 ps, respectively. The Reaction-field method was used for the treatment of the long-range electrostatic interactions. All bonds of the proteins \& peptides were constrained using LINCS algorithm.

The umbrella sampling simulations were performed to compute the binding affinity of GNPS with the spike. The initial configurations were generated using system simulated for $1.5 \mu \mathrm{s}$. The spike protein was slowly pulled from the GNP at a constant speed of $0.01 \mathrm{~nm} / \mathrm{ps}$. Whenever the distance between the center of the mass of RBD and peptide changed by 0.2 $\mathrm{nm}$, that configuration was kept for further simulations. The force constant of $1000 \mathrm{~kJ} \mathrm{~mol}^{-1}$ $\mathrm{nm}^{-2}$ was used to keep the spike restrained at its respective position. Each extracted configuration was simulated for $0.2 \mu \mathrm{s}$ and was used for the potential of mean force calculation. The potential of mean force was generated using the weighted histogram analysis method (WHAM) [41].

\section{Results \& Discussion}

\subsection{Stability of peptide coated GNPs}

\section{1. 1 Stability of peptides bound to GNPs in aqueous solution}

Three different sized nanoparticles $(3,6$ and $10 \mathrm{~nm})$ were used in this study. At first, we performed functionalization of GNPs with peptides and checked their stability because the shape (secondary structure) of the peptide is important for its binding to the RBD of virus spike protein. Six peptides were attached to the GNP of diameter $6 \mathrm{~nm}$ through a thiol bond. Thiols are generally used for functionalizing GNPs due to the excellent affinity of gold towards 
thiol groups [42]. The system was solvated, neutralized, minimized and subsequently equilibrated. The equilibrated structure of peptide coated GNPs in aqueous solution was analyzed. We found that after $0.3 \mu$ s equilibration, the peptides cover-up the GNP nicely as shown in Figure 3(a). Similar functionalization of GNP was also done with 12 peptides as shown in Figure $\mathbf{3}(\mathbf{b})$. We conclude that the peptides are stable in solution and spreads across the NP surface nicely resulting in not only good functionalization of the NP but also aid in stabilization of the nanoparticle. Similar results were obtained for 6 and $10 \mathrm{~nm}$ GNP systems and results of the same are shown in Figure S1 and Figure S2 respectively (please see supporting information section S1).

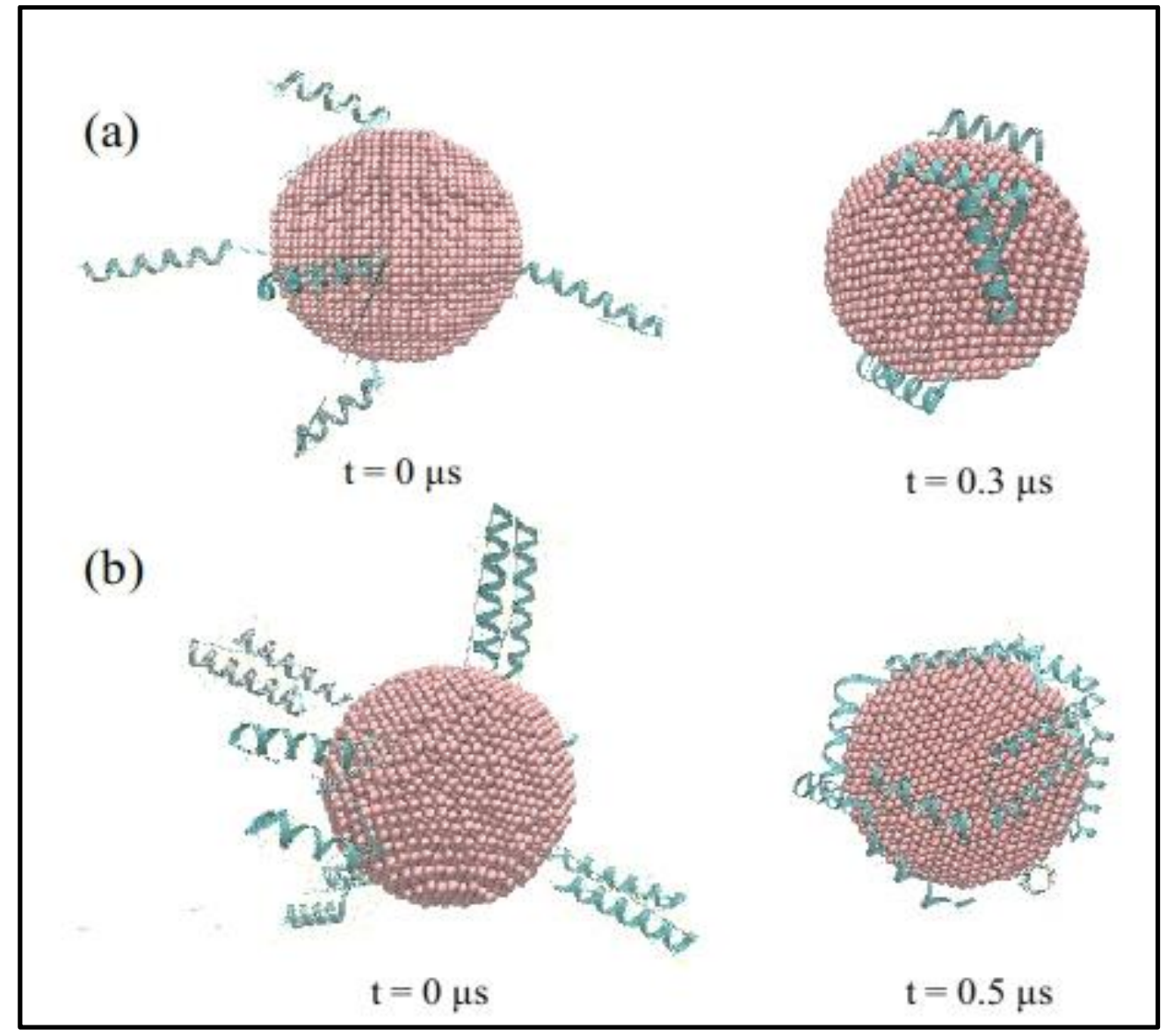

Figure 3: Peptide stability study of GNPs of diameter $6 \mathrm{~nm}$ coated with (a) 6 peptides and (b) 12 peptides. The peptides retain their secondary structure. (color scheme: maroon-GNP, cyan-peptide) (All simulations are done in aqueous solution. water beads are not shown for clarity)

\section{1. 2 Aggregation study of peptide coated GNPs in aqueous solution}

Nanoparticles (NPs), owing to their small size have very high surface energy and tend to agglomerate in solution. As aggregation leads to change in plasmon response, for our study, 
we want the GNPs to aggregate due to binding with the spike and not on their own. So, the GNPs need to be stable (no aggregation in the absence of spike protein) in the solution. Functionalization of GNP with peptide can counter this. Peptide in our study has negative charge and peptide coated GNPs repel each other due to this charge. Thus, aggregation depends on whether this repulsion is strong enough to overcome the attraction due to high energy.

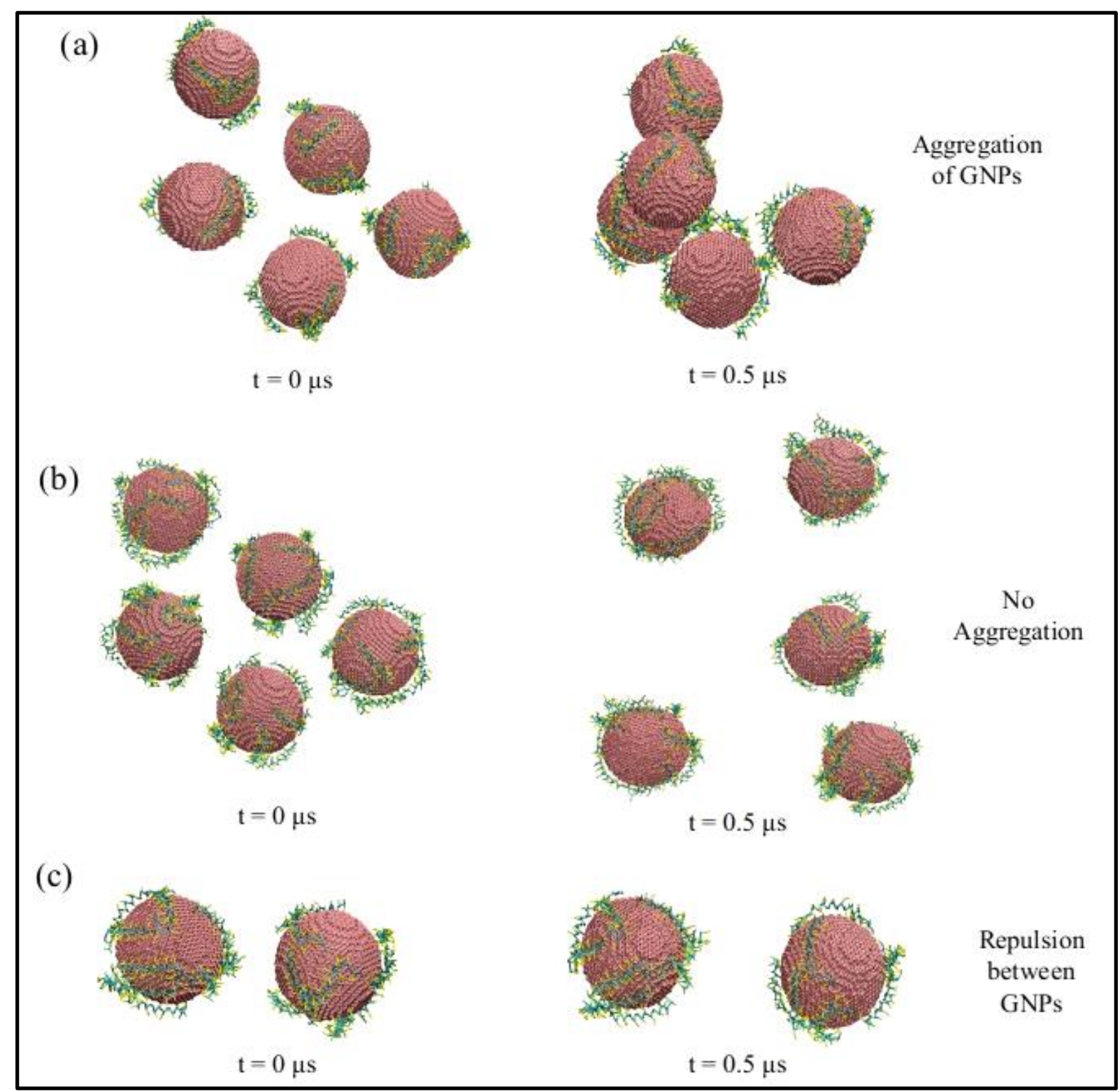

Figure 4: Aggregation study of GNPs of diameter $6 \mathrm{~nm}$ coated with (a) 6 peptides and (b)(c) 12 peptides. 6 peptide coated GNPs agglomerated whereas 12 peptide coated GNPs repel each other. (color scheme: maroon-GNP, cyan/yellow-peptide) (All simulations were performed in aqueous solution. water beads are not shown for clarity) 
In Figure 4, we have observed the stability of peptide coated GNPs of $6 \mathrm{~nm}$ diameter in aqueous solution. It is found that, when 6 peptides are coated on a single GNP, the GNPs in solution tend to agglomerate (Figure 4(a)) whereas when 12 peptides are coated on a single GNP (Figure 4(b)), the GNPs are stable. This is further studied by simulating two 12 peptide coated GNP in solution. From (Figure 4(c)), we observe that there is repulsion between two GNPs leading to no agglomeration.

Similar study was performed for GNP of diameter $3 \mathrm{~nm}$, where it was found that the system is stable in aqueous solution when the GNP is coated with 4 peptides (see supplementary Figure S3). Increasing the size of GNP to $10 \mathrm{~nm}$, we have found that at least 46 peptides are required to be coated on GNP for the system to be stable in aqueous solution (supplementary Figure S4).

Table 1: Minimum peptide density required to stabilize GNP solution for different sizes of GNPs.

\begin{tabular}{|c|c|c|}
\hline $\begin{array}{c}\text { GNP Diameter } \\
(\mathrm{nm})\end{array}$ & $\begin{array}{c}\text { Minimum no. of peptide required to } \\
\text { stabilize GNP solution }\end{array}$ & $\begin{array}{c}\text { Peptide density (No. of peptide } \\
\text { per nm² of GNP surface) }\end{array}$ \\
\hline 3 & 4 & 0.14 \\
\hline 6 & 12 & 0.11 \\
\hline 10 & 46 & 0.15 \\
\hline
\end{tabular}

From stability analysis of peptide coated GNPs of different sizes, we conclude that minimum peptide density required for stable GNP solution lies in the range of 0.11-0.15 peptides $\mathrm{nm}^{-2}$ as summarized in Table 1. Peptide density obtained here can be adopted as basis for coating this peptide on GNPs of higher sizes for experimental study.

In this section, we have discussed that GNPs can easily be functionalized with peptides and stability of these coated nanoparticle depends upon the particle size and the surface density of the peptides. Further, we have studied the interaction of the spike protein of SARS-CoV-2 with peptide coated GNPs. 


\subsection{Interaction of spike protein}

\subsection{Interaction between RBD spike protein and peptide coated GNP}

In the previous section, we investigated the stability of peptide coated GNPs in solution. In this section, we have investigated the binding interaction between peptide coated GNP and RBD protein (RBD taken from PDB ID:6M0J). For this, the RBD protein was placed around $\mathbf{2} \mathrm{nm}$ away from the peptide coated GNP (size $6 \mathrm{~nm}$, no. of peptides on a GNP = 12) and the system was simulated for $1.5 \mu \mathrm{s}$ as shown in Figure $5(\mathrm{a})$. From the analysis of the trajectory, we found that, RBD interacted with the peptide directly and strongly bound to it. Figure 5

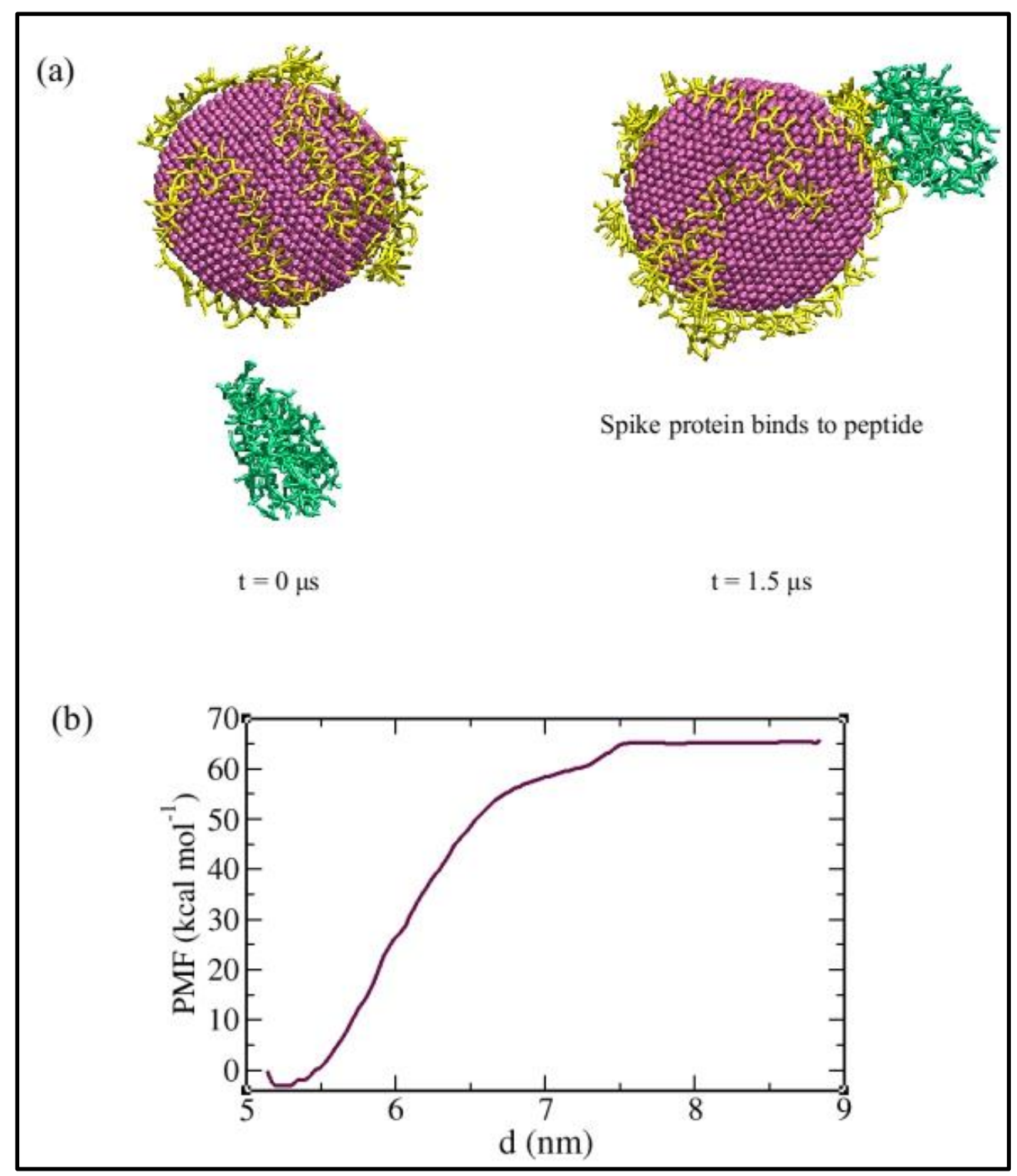

Figure 5: (a) Initial and final configuration of the system simulated for $1.5 \mu \mathrm{s}$. Spike protein was kept $2 \mathrm{~nm}$ away from the peptide coated GNP ( ${ }^{\sim} 6 \mathrm{~nm}$, no of peptides 12 ) at $t=0$. (b) Potential of mean force (PMF) or free energy of RBD in complex with peptide coated GNP. PMF gives the free energy for 
binding of spike and $d$ is the distance between COM of peptide coated GNP and spike protein. (color scheme: purple-GNP, yellow-peptide green-spike RBD protein) (All simulations are done in aqueous solution. water beads are not shown for clarity)

To confirm above findings, we have also performed umbrella sampling simulations to obtain the free energy of the binding interaction between RBD and peptide. The details of the simulations can be found in the Materials and Methods section. The free energy profile is shown in Figure 5(b). The binding free energy of RBD-peptide coated GNP complex was found to be $-66 \mathrm{kCal} \mathrm{mol}^{-1}$. This indicates strong binding between RBD protein and peptide $[43,44]$. Similar study was performed for different configurations of RBD and peptide coated GNP, all resulted in RBD binding to peptide instead of directly interacting with GNP. From this we conclude that the RBD prefers to bind strongly to the peptide coated on GNP. More details on umbrella sampling and free energy calculations can be found in the supplementary section S3. Please note, here we have used GNP of $6 \mathrm{~nm}$ for computational ease, however as shown in Table 1, bigger nanoparticle can easily be used as well with minimum surface peptide density.

\section{2. 2 Interaction between peptide coated GNP and spike protein in bilayer}

In the previous section, we have used the RBD of spike protein to study the interaction between spike protein and peptide coated GNP which gives a good overview of how peptide binds to spike protein. As per Figure 1c, the peptide functionalized GNPs must bind on the virus envelope. In order to mimic the real case scenario, simulation with full spike protein would be more meaningful. Hence, in this section, we have studied the interaction of peptide coated GNP with full-length homo-trimeric spike protein in viral membrane (bilayer). The atomistic structure of full spike protein in viral membrane was taken from Woo et al. [45]. This structure was further coarse-grained using MARTINIZE script [46]. The RBD of spike protein in this structure is between residues 318-541 [45]. For binding analysis, the peptide coated GNP was kept $\sim 1 \mathrm{~nm}$ away from the spike protein and the system was simulated in aqueous environment as shown in Figure 6. 


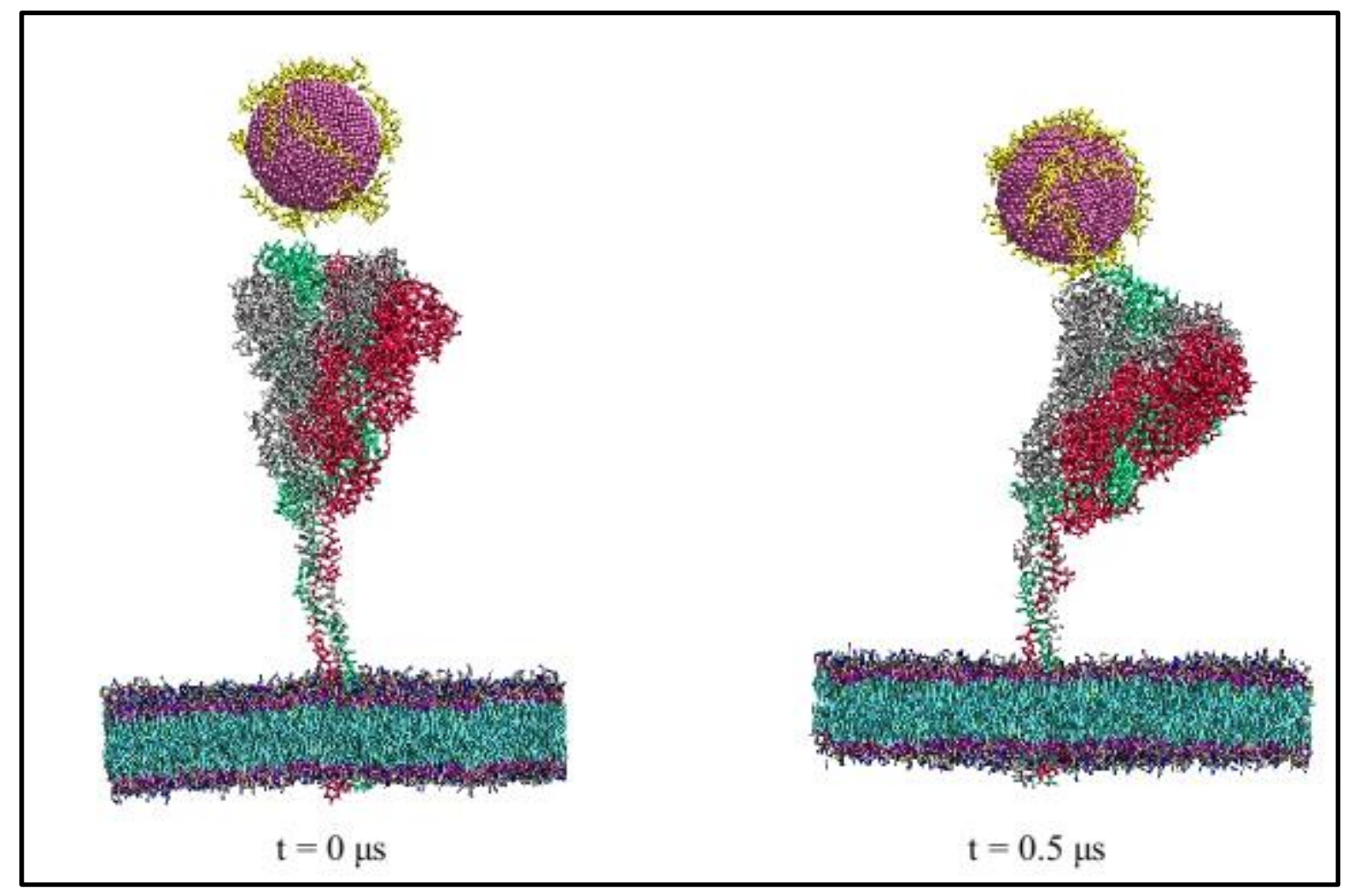

Figure 6: Initial and final configuration of the system simulated for $0.5 \mu \mathrm{s}$. Peptide coated GNP ( $D^{\sim} 6 \mathrm{~nm}$, no. of peptides=12) was kept $1 \mathrm{~nm}$ away from spike protein in bilayer. (color scheme-purpleGNP, yellow-peptide, grey/green/red-three chains of spike protein, cyan/blue-lipids) (All simulations are done in aqueous solution. water beads are not shown for clarity)

From Figure 6, we observe that the spike protein binds to the peptides coated on the GNP. We also note that the peptide interacts with the residue of the spike protein in RBD range of S1 subunit [45]. This conforms to the literature findings reporting RBD interaction with ACE2 receptor $[17,18]$ as the peptide used here is derived from ACE2 protein [32]. This further supports our proposal to use peptide coated GNPs in biosensors for COVID-19 detection.

Further study would require simulation of entire virus or virus like particle (VLPs) in the presence of multiple peptide coated GNPs, to check whether the inter-particle distance between various GNPs is within the range for colorimetric detection. Unfortunately, it is difficult to perform in-silico study for such a large system but similar detection methodology (in-vitro detection of SARS-CoV-2 virus) has been demonstrated recently [29].

Ventura et al. has reported recently an in-vitro study on colorimetric test for fast detection of SARS-CoV-2 based on antibody functionalized GNPs targeting the three surface proteins $(S, E$ and $M$ protein) of the virus [29]. It was reported to be highly effective, sensitive and accurate 
for SARS-CoV-2 detection. This further backs the effectiveness and potential of our proposed technique. The above reported study required identification and preparation of antibodies for specific targeting of viral proteins and owing to larger size of antibodies, the steric hindrance on functionalized GNPs could limit the viral detection. In comparison, the peptide used in our study is much easier to prepare and is also much smaller. Our peptide has played the dual role of functionalizing the GNPs and targeting S protein without UV activation as required by antibody for detection of the virus. Also, in the above-mentioned study [29], excess of antibody is required to ensure full surface coverage whereas our study provides a guidance to use the peptides judicially based on their surface coverage and the size of GNPs. Thus, our proposed method is much simpler in application and may be more suitable for large scale production.

\section{Conclusion}

Like most viruses, several proteins of SARS-CoV-2 are involved in the viral spread and regulate several critical tasks such as the fusion of virus to cell membrane, translation and transcription of viral RNA to name few. Each of these proteins are possible targets for developing effective therapeutics. In this study, we have utilized the preferential binding of the S protein of SARSCoV-2 to the peptide screened from ACE2 receptor of the host cells and proposed a possible method for colorimetric detection based on plasmonic nature of GNPs.

At first, we have functionalized the GNP with potential peptide obtained from Badhe et al. [32] and analyzed their structural stability through molecular simulations. The peptide has retained its secondary structure essential for binding to spike protein. Then, minimum peptide density required for GNPs of different sizes to prevent agglomeration was investigated. After stabilizing peptide coated GNP in solution, we have studied the interaction of spike protein with peptide coated GNP and found that the spike protein interacts preferentially with the peptides as proposed and thus, can be utilized in biosensors.

Further study requires simulation of a much larger system including the entire virus in the presence of multiple peptide coated GNPs, to demonstrate and confirm the effectiveness of our proposed detection tool. Unfortunately, it is difficult to perform in-silico study for such a large system but a similar detection methodology reported by Ventura et al. for in-vitro detection study of SARS-CoV-2 virus [29] supports the potential of our proposed technique. 
In summary, the peptide coated GNPs have potential for possible use in detection of the spike protein of the virus. We have tested these possibilities through in-silico route.In-vitro experimentation of similar systems reported in the literature further supports their potential in clinical biosensing applications.

\section{Acknowledgements}

Authors would like to thank:

- Mr. K Ananth Krishnan, Corporate Technology Officer, Tata Consultancy Services and Dr Gautam Shroff, Head of Research, Tata Consultancy Services for their constant encouragement and support during this project

\section{Funding}

This research was funded by Tata Consultancy Services (TCS), CTO organization. Grant number, 1009292

\section{Authors Contribution}

Nitu Verma, Rakesh Gupta, Auhin Maparu and Beena Rai conceptualized the idea. Nitu Verma, Yogesh Badhe and Rakesh Gupta have performed simulations. Nitu Verma and Yogesh Badhe have analyzed the results. All the authors contributed in discussing the results and writing and editing the manuscript.

\section{References}

1. WHO Coronavirus Disease (COVID-19) Dashboard. https://covid19.ho.int/

2. Cucinotta D, Vanelli M (2020) WHO declares COVID-19 a pandemic. Acta Biomed 91:157-160

3. Moitra P, Alafeef M, Alafeef M, et al (2020) Selective Naked-Eye Detection of SARSCoV-2 Mediated by N Gene Targeted Antisense Oligonucleotide Capped Plasmonic Nanoparticles. ACS Nano 14:7617-7627

4. Zhou P, Yang X Lou, Wang XG, et al (2020) A pneumonia outbreak associated with a new coronavirus of probable bat origin. Nature 579:270-273 
5. Jaiswal G, Kumar V (2020) In-silico design of a potential inhibitor of SARS-CoV-2 S protein. PLoS One 15:1-15

6. Zheng J (2020) SARS-coV-2: An emerging coronavirus that causes a global threat. Int J Biol Sci 16:1678-1685

7. Zhang $\mathrm{H}$, Penninger JM, Li Y, et al (2020) Angiotensin-converting enzyme 2 (ACE2) as a SARS-CoV-2 receptor: molecular mechanisms and potential therapeutic target. Intensive Care Med 46:586-590

8. Wan Y, Shang J, Graham R, et al (2020) Receptor Recognition by the Novel Coronavirus from Wuhan: an Analysis Based on Decade-Long Structural Studies of SARS Coronavirus. J Virol 94:1-9

9. Gheblawi M, Wang K, Viveiros A, et al (2020) Angiotensin-Converting Enzyme 2: SARSCoV-2 Receptor and Regulator of the Renin-Angiotensin System: Celebrating the 20th Anniversary of the Discovery of ACE2. Circ Res 1456-1474

10. Hoffmann M, Kleine-Weber H, Schroeder S, et al (2020) SARS-CoV-2 Cell Entry Depends on ACE2 and TMPRSS2 and Is Blocked by a Clinically Proven Protease Inhibitor. Cell 181:271-280.e8

11. Yi C, Sun X, Ye J, et al (2020) Key residues of the receptor binding motif in the spike protein of SARS-CoV-2 that interact with ACE2 and neutralizing antibodies. Cell Mol Immunol 17:621-630

12. Li F (2016) Structure, Function, and Evolution of Coronavirus Spike Proteins. Annu Rev Virol 3:237-261

13. Cascella M, Rajnik M, Cuomo A, et al (2020) Features, evaluation and treatment of coronavirus (COVID-19). Starpearls

14. Zhu N, Zhang D, Wang W, et al (2020) A Novel Coronavirus from Patients with Pneumonia in China, 2019. N Engl J Med 382:727-733

15. Wu C, Liu Y, Yang Y, et al (2020) Analysis of therapeutic targets for SARS-CoV-2 and discovery of potential drugs by computational methods. Acta Pharm Sin B 10:766-788

16. Vlachakis D, Papakonstantinou E, Mitsis T, Pierouli K (2020) Molecular mechanisms of 
the novel coronavirus SARS-CoV-2 and potential anti-COVID19 pharmacological targets since the outbreak of the pandemic

17. Tai W, He L, Zhang X, et al (2020) Characterization of the receptor-binding domain (RBD) of 2019 novel coronavirus: implication for development of RBD protein as a viral attachment inhibitor and vaccine. Cell Mol Immunol 17:613-620

18. Lan J, Ge J, Yu J, et al (2020) Structure of the SARS-CoV-2 spike receptor-binding domain bound to the ACE2 receptor. Nature 581:215-220

19. Yan R, Zhang Y, Li Y, et al (2020) Structural basis for the recognition of SARS-CoV-2 by full-length human ACE2. Science (80- ) 367:1444-1448

20. Lim Y, Ng Y, Tam J, Liu D (2016) Human Coronaviruses: A Review of Virus-Host Interactions. Diseases 4:26

21. Seo G, Lee G, Kim MJ, et al (2020) Rapid Detection of COVID-19 Causative Virus (SARSCoV-2) in Human Nasopharyngeal Swab Specimens Using Field-Effect Transistor-Based Biosensor. ACS Nano 14:5135-5142

22. Udugama B, Kadhiresan P, Kozlowski HN, et al (2020) Diagnosing COVID-19: The Disease and Tools for Detection. ACS Nano 14:3822-3835

23. Freeman WM, Walker SJ, Kent VE (1975) Quantitative RT-PCR: Pitfalls and Potential. Arzneimittel-Forschung/Drug Res 25:1460-1463

24. Xiao SY, Wu Y, Liu H (2020) Evolving status of the 2019 novel coronavirus infection: Proposal of conventional serologic assays for disease diagnosis and infection monitoring. J Med Virol 92:464-467

25. Bernheim A, Mei X, Huang M, et al (2020) Chest CT findings of coronavirus disease 2019 (COVID-19): Relationship to Duration of Infection. Radiology 30:200463

26. Lamb LE, Bartolone SN, Ward E, Chancellor MB (2020) Rapid Detection of Novel Coronavirus (COVID19) by Reverse Transcription-Loop-Mediated Isothermal Amplification. medRxiv

27. Kellner MJ, Koob JG, Gootenberg JS, et al (2019) SHERLOCK: nucleic acid detection with CRISPR nucleases. Nat Protoc 14:2986-3012 
28. Zhang W, Du RH, Li B, et al (2020) Molecular and serological investigation of 2019-nCoV infected patients: implication of multiple shedding routes. Emerg Microbes Infect 9:386-389

29. Ventura B Della, Cennamo M, Minopoli A, et al (2020) Colorimetric Test for Fast Detection of SARS-CoV-2 in Nasal and Throat Swabs. ACS sensors 5:3043-3048

30. Niikura K, Nagakawa K, Ohtake N, et al (2009) Gold nanoparticle arrangement on viral particles through carbohydrate recognition: A non-cross-linking approach to optical virus detection. Bioconjug Chem 20:1848-1852

31. Zheng L, Wei J, Lv X, et al (2017) Detection and differentiation of influenza viruses with glycan-functionalized gold nanoparticles. Biosens Bioelectron 91:46-52

32. Badhe Y, Gupta R, Rai B (2020) In Silico Design of Peptides with Binding to the Receptor Binding Domain (RBD) of the SARS-CoV-2 and Their Utility in Bio-Sensor Development for SARS-CoV-2 Detection

33. Abraham MJ, Murtola T, Schulz R, et al (2015) Gromacs: High performance molecular simulations through multi-level parallelism from laptops to supercomputers. SoftwareX 1-2:19-25

34. Hess B, Kutzner C, Van Der Spoel D, Lindahl E (2008) GRGMACS 4: Algorithms for highly efficient, load-balanced, and scalable molecular simulation. J Chem Theory Comput 4:435-447

35. Pronk S, Páll S, Schulz R, et al (2013) GROMACS 4.5: A high-throughput and highly parallel open source molecular simulation toolkit. Bioinformatics 29:845-854

36. Humphrey W, Dalke A, Schulten K (1996) VMD: Visual Molecular Dynamics. J Mol Graph $14: 33-38$

37. Monticelli L, Kandasamy SK, Periole X, et al (2008) The MARTINI coarse-grained force field: Extension to proteins. J Chem Theory Comput 4:819-834

38. Lin J, Zhang H, Chen Z, Zheng Y (2010) Penetration of lipid membranes by gold nanoparticles: Insights into cellular uptake, cytotoxicity, and their relationship. ACS Nano 4:5421-5429 
39. Lin JQ, Zhang HW, Chen Z, et al (2011) Simulation study of aggregations of monolayerprotected gold nanoparticles in solvents. J Phys Chem C 115:18991-18998

40. Gupta R, Rai B (2017) Effect of Size and Surface Charge of Gold Nanoparticles on their Skin Permeability: A Molecular Dynamics Study. Sci Rep 7:1-13

41. Kumar S, Rosenberg JM, Bouzida D, et al (1992) THE weighted histogram analysis method for free-energy calculations on biomolecules. I. The method. J Comput Chem 13:1011-1021

42. Liu S, Lämmerhofer M (2019) Functionalized gold nanoparticles for sample preparation: A review. Electrophoresis 40:2438-2461

43. Froloff N, Windemuth A, Honig B (1997) On the calculation of binding free energies using continuum methods: Application to MHC class I protein-peptide interactions. Protein Sci 6:1293-1301

44. Wang J, Alekseenko A, Kozakov D, Miao Y (2019) Improved Modeling of Peptide-Protein Binding Through Global Docking and Accelerated Molecular Dynamics Simulations. Front Mol Biosci 6:1-10

45. Woo H, Park SJ, Choi YK, et al (2020) Developing a fully glycosylated full-length SARSCOV-2 spike protein model in a viral membrane. J Phys Chem B 124:7128-7137

46. De Jong DH, Singh G, Bennett WFD, et al (2013) Improved parameters for the martini coarse-grained protein force field. J Chem Theory Comput 9:687-697 